EPJ Web of Conferences 73, 07002 (2014)

DOI: $10.1051 /$ epjconf/20147307002

(C) Owned by the authors, published by EDP Sciences, 2014

\title{
The MEG experiment at PSI
}

\author{
M. De Gerone ${ }^{\mathrm{a}}$ on behalf of the MEG Collaboration
}

Istituto Nazionale di Fisica Nucleare Sezione di Genova, via Dodecaneso 33, 16146 Genova, Italy

\begin{abstract}
We present the latest results on the lepton flavor violating decay $\mu \rightarrow e \gamma$ based on an analysis of the data collected by the MEG detector at the Paul Scherrer Institute during the years 2009-2011. The analysis of the combined data set, corresponding to $3.6 \times$ $10^{14}$ muons stopped on target provides a $90 \%$ C.L. upper limit of $5.7 \times 10^{-13}$ on the $\mu \rightarrow e \gamma$ branching ratio, constituting the most stringent limit on the existence of this decay to date. After having successfully completed the 2012 and 2013 runs, an upgrade of the experiment, already approved by founding agencies and host laboratory, is foreseen. The status of the R\&D, which has been started since 2012, will be also discussed.
\end{abstract}

\section{Introduction}

The $\mu^{+} \rightarrow e^{+} \gamma$ decay is forbidden in the Standard Model (SM) by the lepton flavor conservation rule. Also including neutrinos mixing, the corresponding Branching Ratio (BR) results to be negligible $\left(<10^{-52}\right)$. Nevertheless, most of the theories proposed as SM extensions foreseen a much larger BR. As an example, in [1] the $\operatorname{BR}\left(\mu^{+} \rightarrow e^{+} \gamma\right)$ is predicted in the range $10^{-12} \div 10^{-14}$. The aim of the MEG experiment is to reach such a sensitivity. The current upper limit (UL) on the $\mu^{+} \rightarrow e^{+} \gamma$ decay, $\mathrm{BR}\left(\mu^{+} \rightarrow e^{+} \gamma\right)<2.4 \times 10^{-12}$ was set by MEG with an analysis based on data taken in the years 20092010 [2]. Here, the improved UL obtained on 2009-2011 combined data sample is presented.

\section{The MEG experiment at PSI}

\subsection{Signal and background}

The event signature of a $\mu \rightarrow e \gamma$ decay at rest is a two bodies final state, with a positron and a photon emitted in time coincidence, moving collinearly back-to-back with their energies equal to half the muon mass $\left(m_{\mu} / 2=52.8 \mathrm{MeV}\right)$. There are two major background sources [3]. The first one comes from the physical background originating by the radiative muon decay $\mu^{+} \rightarrow e^{+} v_{e} \bar{v}_{\mu} \gamma$ (RMD), the other one is an accidental coincidence of a positron from a Michel decay, $\mu^{+} \rightarrow e^{+} v_{e} \bar{v}_{\mu}$, with a high energy photon, coming from RMD, bremsstrahlung or $e^{+}$annihilation in flight. While the RMD rate is proportional to the muon rate $R_{\mu}$, the accidental background grows as $R_{\mu}^{2}$, being the limiting factor of the experiment.

\footnotetext{
a e-mail: degerone@ge.infn.it
}

This is an Open Access article distributed under the terms of the Creative Commons Attribution License 4.0, which permits unrestricted use, distribution, and reproduction in any medium, provided the original work is properly cited. 
Thus, usage of a continuos $\mu$ beam and implementation of a detector with cutting edge resolutions are mandatory.

\subsection{Experimental apparatus}

The MEG experiment is running at the Paul Scherrer Institute (Villigen, $\mathrm{CH}$ ) where the world's most intense DC $\mu$-beam (up to $3 \times 10^{8} \mu / \mathrm{s}$ ) is available. The beam is focused to the target region by a system of magnetic lenses, that has also the purpose to reduce the positron contamination. The target consists of a thin plastic foil fixed at the center of a quasi-solenoidal superconductive magnet, called COBRA. The magnetic field provided by COBRA allows the momentum selection of positrons in the $40 \div 55 \mathrm{MeV}$ energy range with cyclotron orbits smaller than $40 \mathrm{~cm}$ of diameter. Moreover, the positron bending radius is independent from the emission angle and the transverse momentum is adiabatically transferred in the longitudinal direction allowing a faster removal of positrons from the spectrometer central section. This minimize the multiple hits of those positrons that are emitted at large angles on the tracking (Drift Chambers, DC) and timing (Timing Counter, TC) detectors, allowing an easier track reconstruction and a higher detector efficiency. While all the positrons are bounded inside the magnet volume, the emitted photons pass through the thin magnet wall and reach the liquid Xenon calorimeter (LXe), that consists of a volume of $\sim 0.9 \mathrm{~m}^{3}$ of liquid Xenon readout by photomultiplier tubes. All the photon kinematic variables can be reconstructed using the LXe PMTs signals only. The trigger tree takes advantage of the information coming from the fast detectors (LXe and TC), with cuts based on timing, direction and energies of the reconstructed particles. Signals from all detector are digitized by a 1.6 GHz sampling chip developed at PSI, based on the Domino Ring Sampler (DRS). An extensive set of periodic calibration was developed in order to carefully measure and monitor the detector response during the whole DAQ time. A sketch of the MEG experimental layout is shown in Fig. 1, while a more detailed description of each detector can be found in [4].

\section{Data analysis, improvements in 2011 and results}

A "blind analysis" technique is employed in order to avoid any possible bias in results. The analysis algorithms are calibrated using the side-bands data sample. Moreover, also the background level in the signal region can be estimated by the analysis of the side-band regions, being the main source of background the accidental one. The number of signal, RMD and accidental events in the signal region is extracted by means of an extended maximum likelihood fit to the five observables defining the event. The fit is performed in the analysis region, defined as $48 \mathrm{MeV}<E_{\gamma}<58 \mathrm{MeV}, 50 \mathrm{MeV}<E_{e}<56 \mathrm{MeV}$, $\left|t_{e \gamma}\right|<0.7 \mathrm{~ns},\left|\theta_{e \gamma}\right|<50 \mathrm{mrad}$ and $\left|\phi_{e \gamma}\right|<50 \mathrm{mrad}$. The likelihood function is defined as follow:

$$
\begin{aligned}
& \mathcal{L}\left(N_{\mathrm{SIG}}, N_{\mathrm{RMD}}, N_{\mathrm{BG}}\right) \\
& =\frac{e^{-N}}{N_{o b s} !} e^{-\frac{\left(N_{\mathrm{RMD}}-\left(N_{\mathrm{RMD}}\right)\right)^{2}}{2 \sigma_{\mathrm{RMD}}^{2}}} e^{-\frac{\left(N_{\mathrm{BG}}-\left\langle N_{\mathrm{BG}}\right)\right)^{2}}{2 \sigma_{\mathrm{BG}}^{2}}} \prod_{i=1}^{N_{\mathrm{obs}}}\left(N_{\mathrm{SIG}} S\left(\vec{x}_{i}\right)+N_{\mathrm{RMD}} R\left(\vec{x}_{i}\right)+N_{\mathrm{BG}} B\left(\vec{x}_{i}\right)\right),
\end{aligned}
$$

where $N_{\mathrm{SIG}}, N_{\mathrm{RMD}}$, and $N_{\mathrm{BG}}$ are the number of signal, RMD and accidental events respectively, $S, R$ and $B$ are their respective probability density functions (PDF) and $\vec{x}_{i}=\left\{E_{\gamma}, E_{e}, t_{e \gamma}, \theta_{e \gamma}, \phi_{e \gamma}\right\}$ is the vector of observables for the $i-t h$ event. $N_{\text {obs }}$ is the total number of events observed in the analysis window and $N=N_{\mathrm{SIG}}+N_{\mathrm{RMD}}+N_{\mathrm{BG}}$. The PDFs for signal, RMD and accidental background are determined as follow:

- $\mathrm{S}$ is given by the product of the PDFs for the five observables, each defined by their corresponding detector resolutions, measured on dedicated calibration runs; 
Table 1. Summary of sensitivities, best estimates and confidence intervals at 90\% C.L. for 2009-2011 data samples.

\begin{tabular}{|c|c|c|c|c|}
\hline Data sample & $\mu$ stopped & Sensitivity & Best estimate & Upper limit \\
\hline $2009-2010$ & $1.75 \times 10^{14}$ & $1.3 \times 10^{-12}$ & $0.09 \times 10^{-12}$ & $1.3 \times 10^{-12}$ \\
2011 & $1.85 \times 10^{14}$ & $1.1 \times 10^{-12}$ & $-0.35 \times 10^{12}$ & $6.7 \times 10^{-13}$ \\
$2009-2011$ & $3.60 \times 10^{14}$ & $7.7 \times 10^{-12}$ & $-0.06 \times 10^{-13}$ & $5.7 \times 10^{-13}$ \\
\hline
\end{tabular}

- $\mathrm{R}$ is the product of the PDF for $t_{e \gamma}$, which is the same as that for the signal, and the PDF for the other correlated variables, obtained by folding the theoretical spectrum with the detector resolutions;

- $\mathrm{B}$ is determined by the product of the background spectra for each variable, measured on the side-bands.

A frequentistic approach with a profile likelihood-ratio ordering [5] is used to compute the confidence intervals on $N_{\text {SIG }}$. The upper limit on the BR is then obtained by normalizing the upper limit on the number of signals to the total number of Michel decays, counted simultaneously with the signal, using the same analysis cuts. Such a normalization scheme has the great advantage to be independent from the instantaneous rate of the beam and nearly insensitive to the positron acceptance and efficiencies of DC. Some improvements were carried out both from the hardware and software point of view during 2011 run. The DAQ efficiency increased from $72 \%$ to $96 \%$ by implementing a multiple buffer readout scheme; a better knowledge of the DC alignment is obtained by using an new optical surveying based in laser tracking. Moreover, the LXe is better calibrated thanks to the new BGO detector used to tag $\gamma-$ ray during the charge exchange calibration run. Also the analysis algorithm were optimized in 2011. The positron reconstruction based on a Kalman filter technique was totally revised with a better modeling of hits and tracks based on the GEANE package. Furthermore, a FFT-based noise filter was developed to reduce electrical noise of DC signal in offline analysis. On the $\gamma$ side, an improvement in waveform analysis allows for a better pile-up identification and rejection. All these new algorithms were applied on 2011 analysis and on the full combined data set (including 2009-2010 re-analysis). The combined 2009-2011 data sample analysis gives the most stringent BR upper limit on $\mu \rightarrow e \gamma$ of $5.7 \times 10^{-13}$ [6]. The $90 \%$ C.L. intervals as well as the best estimate of the branching ratio for each data set are given in table 1, together with the expected sensitivity. This is defined as the 90\% CL upper limit of the BR averaged over a set of toy MC experiment with a background only hypothesis, and it is calculated to be $1.3 \times 10^{-12}, 1.1 \times 10^{-12}$ and $7.7 \times 10^{-13}$ for the 2009-2010, 2011 and 2009-2011 data set respectively. These numbers are consistent with the BR upper limit observed at the side-bands. The systematic uncertainties for the PDF parameters and the normalization factor are taken into account in the calculation of the confidence intervals by fluctuating the PDFs accordingly to their uncertainties.

\section{The MEG upgrade}

MEG will reach its limit sensitivity, around $5 \times 10^{-13}$, with the analysis of the 2012-2013 data sample. An upgrade program of the MEG experiment (MEG II) has been started since 2012 [7], aiming to improve the experiment sensitivity by an order of magnitude, down to $\sim 5 \times 10^{-14}$. In order to reach such a sensitivity, most of the current detector will be re-designed or modified. A sketch of the upgraded detector is showed in Fig. 1, compared to the current MEG detector. The main characteristics of the upgrade program are the following:

1. usage of a higher beam rate;

2. usage of a thinner target for less background in LXe calorimeter;

3. a higher DC granularity to have more point for a better track reconstruction;

4. a shorter path from last DC point to Timing Counter; 


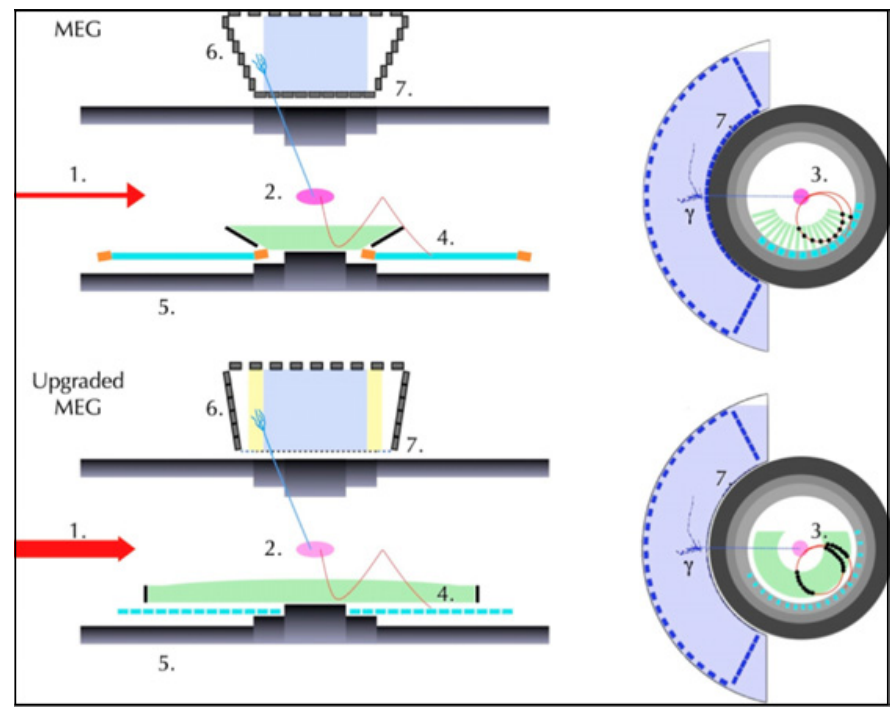

Figure 1. Top: side and front view of the MEG experiment detector layout. Bottom: side and front view of the upgraded MEG experiment detector layout. See text for details.

5. a segmented TC for better matching with DC volume and multiple hit timing method

6. a redesign of the calorimeter shape inside existing cryostat;

7. a higher inner face granularity with new read-out devices (SiPMs) for better energy reconstruction and pile-up rejection.

The MEG II detector is expected to be ready for an engineering run at the end of 2015 .

\section{References}

[1] R. Barbieri et al., Nucl. Phys. B 445, 219 (1995)

[2] J. Adam et al. [MEG Collaboration], Phys. Rev. Lett. 107, 171801 (2011)

[3] Y. Kuno and Y. Okada, Rev. Mod. Phys. 73, 151 (2001)

[4] J. Adam et al., [MEG Collaboration], Eur. Phys. J. C 73, 2365 (2013)

[5] G.J. Feldmann and R.D. Cousins, Phys. Rev. D 57, 3783 (1998)

[6] J. Adam et al., [MEG Collaboration], Phys. Rev. Lett. 110, 201801 (2013)

[7] A. Baldini et al., MEG Upgrade Proposal, [arXiv:1301.7225] [physics .ins-det] 